FTUV 07-0518

\title{
Hidden Dirac Monopoles
}

\author{
Vicente Vento \\ Departamento de Física Teórica and Instituto de Física Corpuscular \\ Universidad de Valencia - Consejo Superior de Investigaciones Científicas \\ 46100 Burjassot (València), Spain, \\ Email: Vicente.Vento@uv.es
}

\begin{abstract}
Dirac showed that the existence of one magnetic pole in the universe could offer an explanation of the discrete nature of the electric charge. Magnetic poles appear naturally in most grand unified theories. Their discovery would be of greatest importance for particle physics and cosmology. The intense experimental search carried thus far has not met with success. I proposed a universe with magnetic poles which are not observed free because they hide in deeply bound monopole-anti-monopole states named monopolium. I discuss the realization of this proposal and its consistency with known cosmological features. I furthermore analyze its implications and the experimental signatures that confirm the scenario.
\end{abstract}

Pacs: 14.80.Hv, 95.30.Cq, 98.70.-f, 98.80.-k

Keywords: nucleosynthesis, monopoles, monopolium 


\section{Introduction}

The theoretical justification for the existence of classical magnetic poles, hereafter called monopoles, is that they add symmetry to Maxwell's equations and explain charge quantization [1]. Dirac showed that the mere existence of a monopole in the universe could offer an explanation of the discrete nature of the electric charge. His analysis leads to the so called Dirac Quantization Condition (DQC),

$$
\frac{e g}{\hbar c}=\frac{N}{2}, \mathrm{~N}=1,2, \ldots,
$$

where $e$ is the electron charge and $g$ the monopole charge [1]. Note that if quarks were asymptotic states the minimum monopole charge would be three times larger.

The origin of monopoles, and therefore their properties, is diverse. In Dirac's formulation monopoles are assumed to exist as point-like particles and quantum mechanical consistency conditions lead to Eq.(1), establishing the value of their magnetic charge. However, their mass, $m$, is a parameter of the theory, limited only by classical reasonings to be $m>2 \mathrm{GeV}$ [2]. In non-Abelian gauge theories monopoles arise as topologically stable solutions through spontaneous breaking via the Kibble mechanism [3]. They are allowed by most Grand Unified Theory (GUT) models, have finite size and come out extremely massive $m>10^{16} \mathrm{GeV}$. Furthermore, there are also models based on other mechanisms with masses between those two extremes [2, 4, 5].

The discovery of monopoles would be of greatest importance not only for particle physics but for cosmology as well. Therefore monopoles and their experimental detection have been a subject of much study since many believe in Dirac's statement[1]

"...one would be surprised if Nature had made no use of it [the monopole]."

At present, despite intense experimental search, there is no evidence of their existence [2, 4, 6, 7, 8]. This state of affairs has led me to investigate a possible mechanism by which monopoles could exist and still be undetectable by present experiments.

Although monopoles symmetrize Maxwell's equations in form there is a numerical asymmetry arising from the DQC, namely that the basic magnetic charge is much larger than the smallest electric charge. This led Dirac himself in his 1931 paper [1] to state,

"... the attractive force between two one quantum poles of opposite sign is $\left(\frac{137}{2}\right)^{2} \approx 4692 \frac{1}{4}$ time that between the electron and the proton. This very large force may perhaps account for why the monopoles have never been separated."

This statement by Dirac has motivated the present investigation. I propose a scenario where monopoles exist but are hidden from our direct observation because 
today they appear forming deep monopole-anti-monopole bound states. I introduce in the next paragraph my proposal which I elaborate in detail in the next sections together with its experimental connotations.

At some early stage in the expansion of the Universe, monopoles and their antiparticles were created. At a later time the dynamics was such that most of the poles paired up to form monopole-anti-monopole bound states called monopolia. This happened because it is easier for the monopoles to interact with each other than with the charged particles in the hot plasma, thus as a consequence also no significant friction force arises. Therefore, the lifetime of the primordial monopolium is solely governed by cascading to the lower bound states where the poles finally annihilate. Moreover, the lifetime of monopolium is sufficiently long to allow primordial monopolia to exist even today in measurable abundances. Thus today, almost all of the existing monopoles, appear confined in deeply bound states [9]. However, the present investigation would be irrelevant if no proof of the existence of monopolium could be found. I analyze signatures of their present existence and of their formation period.

\section{Hidden monopoles}

I envisage a scenario, in which monopoles are not observable as free states at present, which is realized by means of a few assumptions, that satisfies all phenomenological restrictions and leads to new observations which can sustain it. If my scenario is confirmed experimentally it will strongly restrict the way cosmological models deal with monopoles.

At some early stage in the expansion of the universe monopoles and their antiparticles were created by a mechanism which is free from the standard monopole problem [10]. No precise mechanism for their creation is advocated, therefore the mass is not fixed and is left as a parameter to be fitted by consistency requirements. Moreover, monopoles and anti-monopoles existed in the universe, at that time, at the level of abundance compatible with known phenomenological and experimental upper bounds [11, 12, 13]. These are the same starting assumptions of all similar treatments [14, 15, 16]. I next depart from them by assuming that during nucleosynthesis, most of the monopoles and anti-monopoles bind in pairs, due to the strong magnetic forces, to form monopolium. This scheme is realized physically by imposing that: i) the capture radius of the poles, $r_{\text {capture }}$, and the mean free path for charge particle collision in the hot plasma, $\lambda$, satisfy,

$$
r_{\text {capture }}<<\lambda
$$

ii) a consistent monopolium formation scenario. Let me discuss in this section the first assumption which leads to the determination of some of the monopole and monopolium properties and leave for the next section the discussion on monopolium formation. 
The capture radius, $r_{\text {capture }}$, is given by [2, 9],

$$
r_{\text {capture }} \sim \frac{g^{2}}{k T}
$$

The mean free path $\lambda$ is given by

$$
\lambda \sim \frac{1}{\sigma \rho_{c h}},
$$

where $\sigma$ is the cross section for the scattering of the monopole with charged particles [2, 9]

$$
\sigma \sim 2 \frac{m c^{2}}{k T} \text { nanobarns }
$$

$\rho_{c h}$ is the density of charged particles and $m$ is the mass of the monopole.

I describe the monopoles from the monopolium formation era up to the present days by point like Dirac monopoles. It is reasonable to do so since the discussion is largely independent of the detailed structure of the monopoles because it depends only on global properties, i.e., magnetic charge, mass and cosmological abundances.

In the rest of the paper I proceed to show that my assumptions lead to a picture which is consistent with present data.

I describe monopolium as a Bohr atom, with reduced mass $m / 2$ and a strong magnetic, instead of a weak electric, coupling. Its binding energy is

$$
E \sim\left(\frac{1}{8 \alpha}\right)^{2} \frac{m c^{2}}{n^{2}}
$$

where $\alpha=\frac{1}{137}$ is the fine structure constant of $Q E D$ and $n$ the principal quantum number. This equation and those that follow are to be considered only for large principal quantum number $(n>50)$. For low values of $n$ the annihilation mechanism becomes dominant.

The approximate size of the system is given by

$$
r \sim<r>_{n, 0} \sim \frac{12 \hbar}{m c} \alpha n^{2} .
$$

To calculate the mean life I distinguish two processes, i) the cascading process, dominated by dipole radiation [14, which I apply as

$$
\tau_{\text {dipole }} \sim \frac{2 m^{2} c a_{n}^{3}}{\hbar^{2}} \sim 2(12)^{3} \frac{\hbar}{m c^{2}} \alpha^{5} n_{i}^{6},
$$

where $n_{i}$ is the principal quantum number associated with the initial bound state which will be very large $n_{i} \sim 10^{9}$; ii) the annihilation process, which due to the magnitude of $g$ is highly non perturbative and which I next estimate. Looking at the two photon decay process I see [17, 18] 


$$
\tau_{\text {annihilation }}<<\tau_{2 \gamma} \sim 2(4)^{5} \frac{\hbar}{m c^{2}} \alpha^{5} n_{f}^{3},
$$

where $n_{f}$ is the largest principal quantum number associated with a state at which annihilation is still efficient. Since the monopole and anti-monopole only annihilate efficiently when there is a considerable probability of being on top of each other and this only happens for $n<50, n_{f}<<n_{i}$. Thus the annihilation mean life is small compared with the cascading time and can be disregarded in the time scale analysis.

The previous equations can be summarized in terms of the binding energy of the initial bound state and the mean life as,

$$
\begin{aligned}
E_{b}(e V) r_{b}(\AA) & \sim 5.10^{4} e V \AA \\
n_{i} & \sim 9.10^{4}\left[E_{b}(e V)^{1 / 4} \tau(\mathrm{sec})^{1 / 4}\right] \\
m c^{2}(\mathrm{eV}) & \sim 3.10^{7}\left[E_{b}(\mathrm{eV})^{3 / 2} \tau(\mathrm{sec})^{1 / 2}\right] \mathrm{eV}
\end{aligned}
$$

Here $E_{b}$ and $r_{b}$ are respectively the binding energy and the radius of the initial bound state.

Using Eqs. (21) through (12) my scenario can be constructed. I assume that capture takes place for a binding energy slightly higher, to avoid thermal dissociation, than $k T=1 \mathrm{MeV}$,

$$
E_{b}>1 \mathrm{MeV} .
$$

This temperature is not related to the scale for production of monopoles but to that at which monopolium, the bound state, is formed from already existing monopoles [14.

These equations and the temperature scale of the proposed scenario show that monopolium is a very tightly bound system

$$
r_{b}<0.05 \AA<r_{\text {capture }} \sim 0.07 \AA .
$$

Let me now proceed to the calculation of the mean free path,

$$
\lambda \sim \frac{1}{\sigma \rho_{c h}} .
$$

The cross section at $k T=1 \mathrm{MeV}$ is given by

$$
\sigma \sim 2.10^{-23} \frac{m c^{2}(\mathrm{eV})}{\mathrm{eV}} \AA^{2}
$$

From Eq.(12) I have

$$
\frac{m c^{2}(\mathrm{eV})}{\mathrm{eV}}=1.510^{25} \eta^{\frac{1}{2}}
$$


where $\eta=\tau / \tau_{U}, \tau$ being the lifetime of the monopolium and $\tau_{U}$ the age of the universe, which we have taken approximately to be $\tau_{U}=1 / H_{0} \sim 3.10^{17}$ sec.

Thus the cross section becomes

$$
\sigma \sim 3.10^{2} \eta^{\frac{1}{2}} \AA^{2}
$$

I now calculate the density of charged particles. The density of photons is given by [10

$$
\rho_{\gamma}=\frac{2 \zeta(3)(k T)^{3}}{\pi^{2}(\hbar c)^{3}},
$$

while the ratio of the densities of nucleons to photons is given in nucleosynthesis by $\rho_{N} / \rho_{\gamma} \sim 4$. $-7.10^{-10}$. At $k T=1 \mathrm{MeV}$, we have mostly nucleons and since $\rho_{n} / \rho_{p} \sim \exp (-1.293 / k T)$, we obtain

$$
\frac{\rho_{p}}{\rho_{\gamma}} \sim 1 .-3 \cdot 10^{-10}
$$

Due to neutrality for each proton there is an electron, thus

$$
\rho_{c h} \sim 2.510^{22} \text { particles } / \mathrm{cm}^{3} .
$$

Thus putting together Eq.(15) and Eq.(16) I obtain

$$
\lambda \sim 0.1 \eta^{\frac{1}{2} \AA} .
$$

In Fig. 11 I show the ratio of $\lambda$ over $r_{\text {capture }}$ as a function of the life of monopolium in units of the age of the universe. I also plot the ratio of the average distance between charges to $r_{\text {capture }}$. The plot shows that for lifetimes above $0.1 \tau_{U}$ the mean free path is smaller than the mean distance between charges and the capture radius becomes of the order of magnitude of the mean free path. Thus for monopolium lifetimes of the order of the age of the universe the conventional scenarios would take place [14, 15, 16]. However, for $\tau / \tau_{U}<0.1$ the condition Eq.(2) will be satisfied and a completely different scenario occurs whose existence has been a matter of debate since the idea was first proposed [19].

In order to clarify the issue let me recall once more the work of Blanco-Pillado and Olum [16]. They pointed out that in the standard scenario the drag force felt by the monopole in the plasma reduces dramatically the mean life of the state [16] and therefore relic monopolia do not influence present day observations. This is so if we are to the left of $\tau / \tau_{U}>0.1$. However, their phenomenon is not active in the scenario presented here, i.e., when $\tau / \tau_{U}<0.1$ for obvious reasons. As shown in ref. [21] the drag force depends on the two limits of an impact parameter integral,

$$
F_{\text {drag }} \sim \text { coefficient } \int_{b_{\min }}^{b_{\max }} d b / b
$$




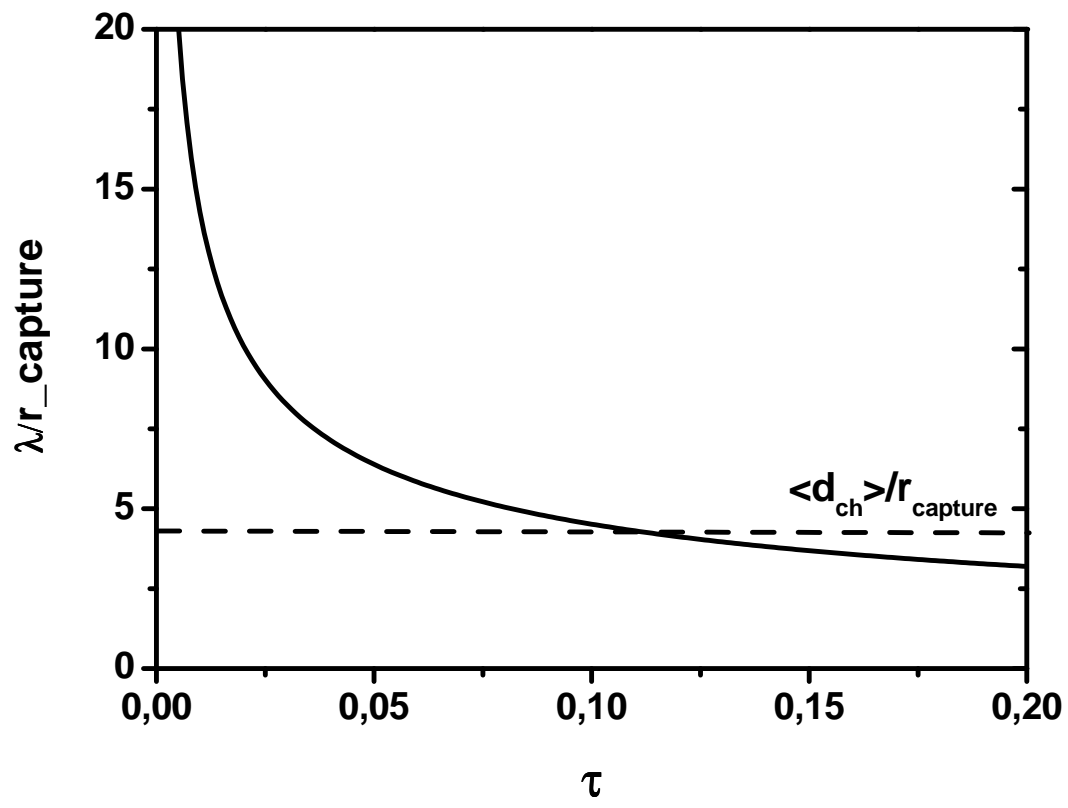

Figure 1: The ratio $\lambda / r_{\text {capture }}$ is presented as a function of monopolium lifetime $\tau$ in units of age of the universe $\tau_{U}$ at $k T=1 \mathrm{MeV}$. Also the ratio of the average distance between charge particles $\left\langle d_{c h}>/ r_{\text {capture }}\right.$ at the same temperature is shown.

where naturally the force only arises if $b_{\min }<b_{\max }$. The small limit has to do with the monopole interaction with the plasma, in our case $b_{\min } \sim \lambda$. The large limit depends on the interaction of the monopoles inside the monopolium with the charge particles and can not extend beyond $r_{\text {capture }}, b_{\text {max }} \sim r_{\text {capture }}$. Due to our assumption Eq.(2), as seen in Fig. 1, $r_{\text {capture }}<\lambda$ in the region of interest and therefore in our scenario there is no drag force. It is interesting to note that the solution that Blanco-Pillado and Olum find to solve the impasse, i.e. monopoles attached by strings [16], reproduce with a complex dynamics the same scenario for monopolium I obtain, i.e. non-relativistic monopoles very closely tight together so that the light charges do not interact with them and they only loose energy by radiation.

\section{Monopolium formation}

In the previous section I have shown that the proposed scenario might be realized if $\eta<<1$ since under these conditions primordial monopolium might be observed today. I here study the other ingredient of the scheme, a plausible scenario for the formation rate of monopolium which gives consistency to the scheme. The scheme is based on three time (temperature) scales. The first time scale is the formation scale of monopoles, $t_{i}$, whose corresponding temperature is $T_{i}$. This temperature is very high, consistent with the large mass of the monopoles determined in the previous section. The second time scale ends at the beginning of the monopolium bound 
state stability period, i.e. when the temperature is lower then the minimum binding energy, i.e. $T_{f} \sim 1 \mathrm{MeV}$. Finally the third time scale is today, i.e. $T_{\text {today }} \sim 2.7^{0} \mathrm{~K}$.

In this section I will study the formation rate of monopolium from the early universe to the beginning of the stability period, i.e. from $T_{i}$ to $T_{f}$. In the next section I will study the decay process to find present day monopolium abundance, i.e. from $T_{f}$ to $T_{\text {today }}$.

Following Blanco-Pillado and Olum [16] one can write the evolution equation as

$$
\frac{d \Gamma}{d T}=-A \gamma^{2}(T)\left(\frac{1}{T}\right)^{(9 / 10)} .
$$

In this equation $\Gamma$ represents the comoving monopolium density, i.e. $N_{M \bar{M}} / s$, where $N_{M \bar{M}}$ is the monopolium density and s the entropy density; $\gamma$ is the monopole comoving density, i.e. $n_{M} / s$, where $n_{M}$ is the monopole density; $T$ is the temperature and $A$ an softly temperature dependent quantity.

Let $\gamma$ depend on temperature as

$$
\gamma=B T^{\delta},
$$

where $\mathrm{B}$ is a constant, a possibility contemplated in ref. [20]. The above equation can be easily solved leading to

$$
\Gamma\left(T_{i}\right)-\Gamma\left(T_{f}\right)=-\frac{A}{2 \delta+0.1}\left(\gamma^{2}\left(T_{i}\right) T_{i}^{0.1}-\gamma^{2}\left(T_{f}\right) T_{f}^{0.1}\right)
$$

My fundamental hypothesis implies,

$$
\begin{aligned}
& \Gamma\left(T_{i}\right)<<\Gamma\left(T_{f}\right), \\
& \gamma\left(T_{i}\right)>>\gamma\left(T_{f}\right) .
\end{aligned}
$$

Therefore,

$$
\Gamma\left(T_{f}\right) \approx \frac{A}{2 \delta+0.1} \gamma^{2}\left(T_{i}\right) T_{i}^{0.1} .
$$

Dividing by $\gamma\left(T_{f}\right)$ I arrive at,

$$
\frac{N_{M \bar{M}}\left(T_{f}\right)}{n_{M}\left(T_{f}\right)} \approx \frac{A B}{2 \delta+0.1} T_{i}^{0.1}\left(\frac{T_{i}^{2}}{T_{f}}\right)^{\delta} .
$$

Thus if $\delta>0$, since $T_{i}>>T_{f}$, the wishful scenario is realized. Note that this behavior corresponds to $p>1$ in ref. [20]. 


\section{Monopolium abundance}

I proceed in here to investigate phenomenological consistencies of the proposed picture. Let us calculate the present abundance of monopolium taking as input the abundance during the formation period. By doing so my aim is to find consistency between the proposed scenario and the observation.

The equation governing the density of monopolia $\rho$ taking into account the expansion of the universe is given by

$$
\dot{\rho}(t)=-\frac{1}{\tau} \rho(t)-3 \frac{\dot{R}}{R} \rho(t),
$$

where $\mathrm{R}$ is the scale factor of the universe and $\dot{A}$ denotes $d A / d t$. If the expansion is adiabatic $(R T \sim$ constant) and the universe is radiation dominated, the expansion rate is given by

$$
\frac{\dot{R}}{R}=-\frac{\dot{T}}{T}=\frac{T^{2}}{\Lambda}
$$

Here, $\mathrm{T}$ is the temperature of the universe and $\Lambda$ a quantity related to the Planck mass and the effective degrees of freedom [20]. The temperature equation in Eq.(24) can be integrated to give

$$
t=\frac{\Lambda}{2 T^{2}}
$$

establishing the relation between evolution time and temperature. Using Eqs.(23), (24) and (25) one obtains,

$$
\dot{\rho}(t)=-\left(\frac{1}{\tau}+\frac{3}{2} \frac{1}{t}\right) \rho(t)
$$

which can be easily integrated giving

$$
\rho(t)=\rho\left(t_{0}\right)\left(\frac{t_{0}}{t}\right)^{3 / 2} \exp \left(-\frac{t-t_{0}}{\tau}\right) .
$$

I now take this equation, which is the conventional equation for the decay of un unstable system in an expanding universe [10], and adapt it to my interests, namely I want to study the evolution of the number of monopolia between $T_{f}$ and $T_{\text {today }}$. Using that, in this case, $t=t_{\text {today }}=\tau_{U}>t_{0}=t\left(T_{f}\right) \sim 1 \mathrm{sec}$, and the relation between time and temperature, Eq. (25), one can write

$$
\rho\left(T_{\text {today }}\right)=\rho\left(T_{f}\right)\left(\frac{T_{\text {today }}}{T_{f}}\right)^{3 / 2} \exp \left(-\frac{\tau_{U}}{\tau}\right) .
$$

In the present situation this equation reduces to

$$
\rho\left(2.7^{0} K\right)=1.2510^{-29} \rho(k T=1 \mathrm{MeV}) \exp \left(-\frac{1}{\eta}\right),
$$




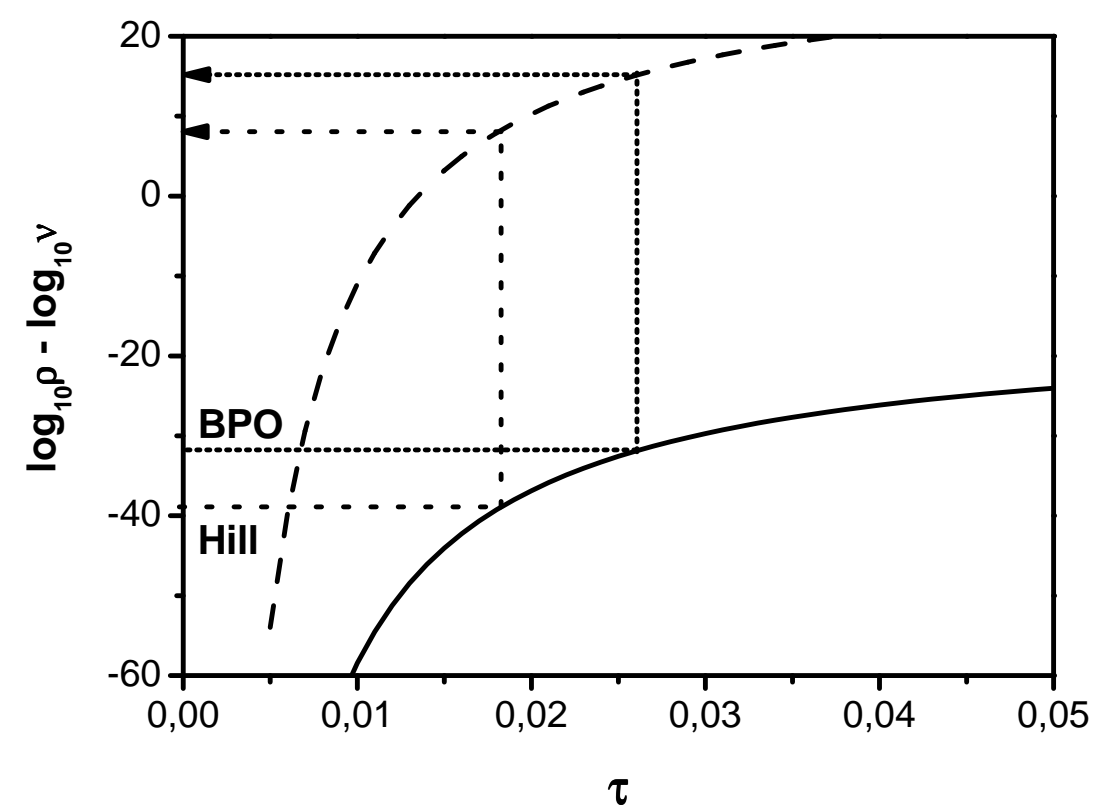

Figure 2: The density in a $\mathrm{cm}^{3}\left(\log _{10}\right)$ of monopolia (solid curve) and the number $\left(\log _{10}\right)$ of monopolia decays in a $p c^{3}$ (dashed) today presented as a function of monopolium lifetime $\tau$ in units of age of the Universe $\tau_{U}$. The values by Hill [14] (dot) and Blanco-Pillado and Olum [16] (short dot) for the density of monopolia are used to extract the number of decays.

where $\eta=\tau / \tau_{U}$.

The standard scenario for helium synthesis requires that the mass of the monopole does not dominate the universe when $k T=1 \mathrm{MeV}$, this implies [20],

$$
\rho_{\text {monopole }}(k T=1 \mathrm{MeV}) \leq 1.310^{14}\left(\frac{m c^{2}(\mathrm{eV})}{\mathrm{eV}}\right)^{-1} \stackrel{\circ}{A}^{-3} .
$$

I assume that the density of monopoles is equal to the density of anti-monopoles and since most of them are bound equal to the density of monopolia, thus one gets from Eq.(29) for the density of monopolia today,

$$
\rho\left(2.7^{0} \mathrm{~K}\right) \sim 1.610^{-15}\left(\frac{m c^{2}(\mathrm{eV})}{\mathrm{eV}}\right)^{-1} \stackrel{\circ}{A}^{-3},
$$

which using Eq. (12) leads to

$$
\rho\left(2.7^{0} \mathrm{~K}\right) \sim 10^{-16} \frac{\exp \left(-\frac{1}{\eta}\right)}{\eta^{3 / 2}} \mathrm{~cm}^{-3} .
$$

This equation establishes a relation between the parameter characterizing the various scenarios $\eta$ and the observation.

Let me calculate the number of decays per year in a given volume of the universe 


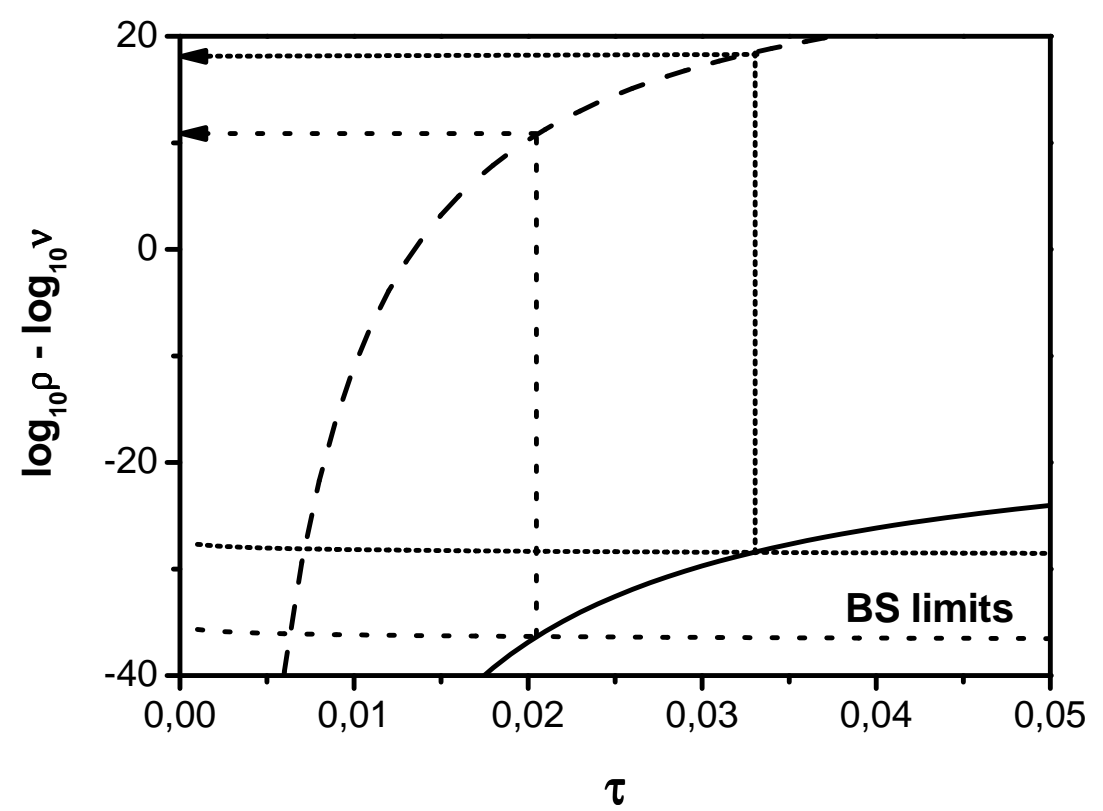

Figure 3: The density in a $\mathrm{cm}^{3}\left(\log _{10}\right)$ of monopolia (solid curve) and the number in a $p c^{3}\left(\log _{10}\right)$ of monopolia decays (dashed) today presented as a function of the monopolium lifetime $\tau$ in units of age of the Universe $\tau_{U}$. The limits of the values for the density of monopolia from the values of Bhattarchee and Sigl [15], $1<\Omega_{M} h^{2}<10^{8}$ are plotted and the corresponding number of decays shown.

$$
\nu=N\left(1-\exp \left(-\frac{1}{\tau}\right)\right) \sim \frac{N}{\tau(\text { years })},
$$

where I have used the approximation that $\tau$ (years) $>>1$ year. Note also that the temperature factor in Eq.(23) drops out because it is very close to $1 . N$ is the number of monopolia in the given volume. Let me choose to calculate the observation a volume of $1(\mathrm{pc})^{3}$, then I get for the number of events in one year

$$
\nu \sim 2.710^{29} \frac{\exp \left(-\frac{1}{\eta}\right)}{\eta^{3 / 2}} .
$$

Monopolium has been associated with Ultra High Energy Cosmic Rays (UHECR) [22, 23 in various schemes [14, 15, 16]. This association leads to a phenomenological determination of its abundance. I look for consistency between the phenomenological determined abundances and the monopolium mean life obtained in the calculation.

In Fig. (2) we show the results of our calculation as a function of $\tau$ in units of $\tau_{U}$ and I introduce two numerical values for the density as obtained by

i) Hill [14]: $\rho\left(2.7^{0} \mathrm{~K}\right) \sim 10^{-39} \mathrm{~cm}^{-3}$

ii) Blanco-Pillado and Olum [16] $: \rho\left(2.7^{0} \mathrm{~K}\right) \sim 10^{-32} \mathrm{~cm}^{-3}$ 
In Fig.(3) I compare my results to bands, characterized by $1<\Omega_{M} h^{2}<10^{8}$, obtained from the density equation of Bhattacharjee and Sigl [15].

The above study shows that proposed values for the density of monopolia are consistent with $\eta \sim 0.02$ and therefore with

$$
\lambda \sim 10 r_{\text {capture }}
$$

If we look at monopolia decays we realize that their number is

$$
10^{6}<\nu<10^{18} \text { decays } / \mathrm{pc}^{3}
$$

which is large compared with those obtained in other models.

Please note that all the estimates are based on the assumption that all UHECRs are due to monopolia. If they are additional mechanisms for UHECRs formation or if the number of observations [22, 23] diminish, the density of monopolia today would decrease and the proposed scenario would become more natural. However, this would also imply that it would be harder to confirm experimentally.

I conclude from the above analysis that at present most monopoles in the intergalactic vacuum are bound in deep bound states $n \sim 50$ close to the annihilation levels and therefore their binding energy is at the level of

$$
E_{b} \sim 10^{14} \mathrm{GeV}
$$

supporting Dirac's conjecture for the non observability of monopoles.

Moreover, by looking back at Eq.(12) one sees that

$$
m c^{2} \sim 2.10^{15} \mathrm{GeV},
$$

which is very large and comparable to the values arising from GUT models.

\section{Monopolium detection}

Ideally we would like to be able to produce the monopolium in our laboratory. Its mass $M c^{2} \sim 10^{15} \mathrm{GeV}$ makes laboratory production impossible. Could we capture a monopolium in our laboratory and measure its properties? It soon will become clear this is an impossible task. Monopolium is a sterile particle under laboratory conditions.

The present day background monopolium density is small.

$$
\rho\left(2.7^{0} \mathrm{~K}\right)<10^{-32} \mathrm{~cm}^{-3}
$$

At present monopolia are mostly thermal and therefore their velocity is of the order

$$
v \sim 10^{-6} \mathrm{~m} / \mathrm{sec}
$$


Thus the average number of particles in our detector would be

$$
N_{M} \sim \rho v t A<10^{-19} \text { (t/years) }\left(A / K m^{2}\right),
$$

where $A$ is the area of the detector and $t$ the time of exposure. Thus the chances of having one localized event are insignificant.

Let me assume that a monopolium enters by chance a detector, what could we observe? Due to the dual behavior of the Maxwell equations in the presence of monopoles [24, monopolium has an electric dipole moment

$$
\vec{p} \sim \frac{g}{2 m c} \vec{L}
$$

and a magnetic dipole moment

$$
\vec{\mu} \sim 2 g c<\vec{r}>_{l \neq 0}
$$

In the presence of an electric or magnetic field they tend to orient against the field and become effectively

$$
p \sim \frac{g}{2 m c} m_{l}
$$

and

$$
\mu \sim 2 \frac{\hbar^{2} c}{m g}\left(3 n^{2}-l(l+1)\right),
$$

where $n, l, m_{l}$ are respectively the principal, the orbital and the magnetic quantum numbers. Substituting the values of the couplings and masses I get

$$
p \sim 10^{-29} m_{l} \text { (e-charge) meter }
$$

and

$$
\mu \sim 10^{-22}\left(3 n^{2}-l(l+1)\right) \mathrm{eV} / \text { Tesla }
$$

Note that, the maximum achievable electric and magnetic field gradients today , 50 $\mathrm{MV} /$ meter $^{2}$ and 5 Tesla/meter, produce in background monopolia, with $n \sim l \sim$ $m_{l} \sim 100$, insignificantly small energies and forces. It is easy to calculate, that to stop them, moving at thermal velocities, we would need distances and times of universe scales.

Thus I conclude, that from the point of view of traditional laboratory experiments, monopolia are sterile and we have to center our attention in astrophysical observations.

Let me now turn to astrophysical observations. Let me distinguish two periods: the formation period and the immediate past.

During the formation period $n \sim 10^{9}$. Cascading for large values of $n$ leads to Larmor type emission 


$$
\lambda \sim 16 \alpha^{2} \frac{h}{m c} n_{n}^{3} \sim 32 \frac{\mathrm{eV}}{m c^{2}} n^{3} \AA,
$$

thus the wave length during the nucleosynthesis period will be

$$
\lambda \sim \text { milimeter }
$$

Therefore, there should be an isotropic background radio frequencies as a remnant of that period.

In the immediate past even until today, most monopolia are close to the annihilating stage, i.e., $n<100$. The Larmor formula is still approximately applicable, thus

$$
\lambda<10^{-15} \stackrel{\circ}{A},
$$

which implies that the emitted photons will have a huge energy.

$$
h \nu \sim 10^{10} \mathrm{GeV}
$$

This energetic photons will occur at the level of $10^{15}-10^{20}$ per $\mathrm{pc}^{3}$ and therefore should be seen. However, their distribution will not be isotropic. The core of galaxies, and of clusters of galaxies, provide an environment of high electric and magnetic field gradients, thus the small electric and magnetic dipoles of monopolium will change their distribution in these environments and create geographic anisotropies.

Moreover, these regions also provide an environment with high energy and high density where monopolia might be excited to the point of break up. Thus, also the low frequency spectrum will acquire geographical distributions which become anisotropies in the spectrum over the isotropic spectrum remnant of the formation era. Note that very few monopolia can be formed after electron-positron annihilation due to the lack of remnant monopoles and the absence of monopole pair formation due to their huge masses, except in these very energetic environments, and this phenomenon will not affect greatly the calculated density of monopolia [16].

Finally, monopolium can annihilate into UHECRs, at the level of millions per year and per cubic parsec, under present experimental expectations [22, 23] provided it is the only mechanism, depositing a huge amount of energy, $E>10^{15} \mathrm{GeV}$, in a small region of space-time leading to what Hill [14] calls a cataclysmic scenario, whose details depend on the microscopic theory of monopole formation.

\section{Conclusions}

The possibility of having monopoles in nature is appealing. I have presented a scenario for the universe in which relic monopoles still exist today however, not as free particles, but deeply bound in monopolium states. The crucial ingredients of my proposal are: i) that, in the early universe, the mean free path of monopoles is much 
larger than their capture radius, and therefore they bind so tightly in monopolium that they barely interact with the surrounding plasma, surviving in this way the effect of the drag force and only emitting energy by radiation until their annihilation; ii) the evolution of the monopole density is governed by a strongly temperature dependent function leading to a large production of monopolia. Few monopolia are formed in the second period since almost no free monopoles exist, because they are to produce at these low temperatures due to their large mass and strong binding within monopolia, to drive the formation rate. The initial density of monopolia and their lifetime might explain UHECRs by construction.

Three distinctive quantities determine the consistency of the various requirements in the scheme: the monopole evolution parameter $\delta$; the temperature of monopolium formation $(k T \sim 1 \mathrm{MeV})$; and the mean life of the state $\left(\tau \sim 10^{8}\right.$ years). The outcome is monopolium, a neutral particle protected from the interaction with the medium in a strongly bound state, which radiates copiously until ultimately annihilates in a cataclysmic scenario being a possible source UHECRs.

The detection of monopolia, and therefore the existence of monopoles, presents interesting signatures associated with the monopolium spectrum, i.e., a diffuse isotropic radio frequency background remnant of its formation period with geographical anisotropies produced by more recent activities, and a high frequency monopolium spectrum associated with the last period of its lifetime, which manifests itself by anisotropically distributed high energy gamma rays.

\section{Acknowledgments}

I thank T. Sloan for a careful reading of the manuscript. Discussions with Gabriela Barenboim, Jose Bordes, Carlos García-Canal, Huner Fanchiotti, Pedro González, Santiago Noguera and Arcadi Santamaría are acknowledged. This work was supported by MCYT-FIS2004-05616-C02-01 and GV-GRUPOS03/094.

\section{References}

[1] P.A.M. Dirac, Proc. Roy. Soc. A133 (1931) 60, Phys. Rev. 74 (1940) 817.

[2] N. Craigie, G. Giacomelli, W. Nahern and Q. Shafi,Theory and detection of magnetic monopoles in gauge theories, World Scientific, Singapore1986.

[3] T.W.B. Kibble, J. Phys. A 9 (1976) 1387, Phys. Rep. 67 (1980) 183; A. Vilenkin, Phys. Rep. 121 (1985) 263.

[4] G. Giacomelli and L. Patrizii, hep-ex/0506014.

[5] A. De Rújula, Nucl. Phys.B435 (1995) 257.

[6] K. A. Milton, hep-ex/0602040. 
[7] Review of Particle Physics, S. Eidelman et al. Phys. Lett. B592 20041.

[8] M. J. Mulhearn, Ph.D. Thesis MIT 2004.

[9] Ya.B Zeldovich and M. Yu. Khlopov, Phys. Lett. 79 (1978) 239.

[10] E.W. Kolb and M.S. Turner in The Early Universe, Addison-Wesley, New York (1990).

[11] S. Ahlen et al., Phys. Rev. Lett. 72 (1994) 608

[12] E.N. Parker, Astrophys. J. 180 (1970) 383; M.S. Turner, E.N. Parker and T. Bogdan, Phys. Rev. D26 (1982) 1296.

[13] F.C. Adams et al., Phys. Rev. Lett. 70 (1993) 2511.

[14] C.T. Hill, Nucl. Phys. B224 (1983) 469

[15] P. Bhattacharjee and G. Sigl, Phys. Rev. D51 (1995) 4079.

[16] J. J. Blanco-Pillado and K. D. Olum, Phys. Rev. D60 (1999) 083001.

[17] P.A.M. Dirac, Proc. Camb. Phil.Soc. 26 (1930) 361.

[18] J.A. Wheeler, Ann. N.Y. Acad. Sci. 46 (1946) 221.

[19] V. Vento, astro-ph/0511764.

[20] J.P. Preskill, Phys. Rev. Lett. 43 (1979) 1365.

[21] T. Goldman, E.W. Kolb and D. Toussaint, Phys. Rev. D23 (1981) 867.

[22] N. Hayashida et al., Phys. Rev. Lett. 73, 3491 (1994).

[23] D.J. Bird et al., Astrophys. J. 424, 491 (1994).

[24] J. D. Jackson, Classical Electrodynamics, de Gruyter, N.Y. (1982). 